\title{
A preliminary study on the synthetic lethal effect of berberine and olaparib on pancreatic cancer cells and its mechanism
}

\author{
Chengyong Zhang ${ }^{1 \#}$, Tingting Yang ${ }^{1 \#}$, Xiaoting Chen ${ }^{1}$, Jiexuan $\mathrm{Xu}^{1}$, Danlu Liang ${ }^{1}$, Huairu Yi ${ }^{1}$, Size Chen ${ }^{2}$, Lanlan Huang ${ }^{2}$ \\ Naihua Liu ${ }^{2}$, and Shaoqiang Lin $^{1,2^{*}}$ \\ ${ }^{1}$ Clinical Department of Guangdong Metabolic Disease Research Centre of Integrated Chinese and Western Medicine, The First \\ Affiliated Hospital of Guangdong Pharmaceutical University, No.19 Nonglinxia Road, 510000, Guangzhou, China. \\ ${ }^{2}$ Precision Medical Engineering Research Center for Esophageal Cancer of Guangdong Province at The First Affiliated Hospital of \\ Guangdong Pharmaceutical University, No.19 Nonglinxia Road, 510000, Guangzhou, China. \\ ${ }^{\#}$ Contribute equally to this work.
}

\begin{abstract}
Pancreatic cancer is a kind of malignant tumor with high mortality rate. Early operation and late chemoradiotherapy are the treatment criteria, but the prognosis is still poor. Berberine, an alkaloid compound present in many herbal plants, is capable of inducing oxidative DNA damage and downregulating homologous recombination repair (HRR) in cancer cells. Poly (ADP ribose) polymerase-1 (PARP-1) is a sensor of DNA damage with key roles in DNA repair. In this study, we demonstrated that berberine and PARP inhibitor olaparib have a synthetic lethal effect on pancreatic cancer cells. The expression level of RAD51 were reduced by berberine. Correspondingly, PARP became hyperactivated in response to berberine treatment. When berberine is combined with olaparib, the expression of Rad51 and Parp are inhibited. The combination of berberine and olaparib synergistically inhibit cell activity and induce cell apoptosis. In addition, the synergistic effect of berberine and olaparib can be reversed by apoptosis inhibitor and necrosis inhibitor. Together, the results indicate that berberine combined with olaparib have a synthetic lethal effect on pancreatic cancer cells.
\end{abstract}

\section{Effects of berberine and olaparib alone and in combination on cell viability}

As shown in Figure 1, berberine inhibited the proliferation of all four types of pancreatic cancer cells, among which the IC50 value of berberine treated Miapaca-2 cell was $48.546 \pm 3.54 \mu \mathrm{M}$, and the IC50 value of olaparib treated Aspc-1 cell was $34.409 \pm 1.22 \mu \mathrm{M}$. Since pancreatic cancer cells were not sensitive to berberine and olaparib at low concentrations, berberine $(5 \mu \mathrm{M})$ was used in combination with olaparib $(5 \mu \mathrm{M})$. as shown in Figure 2, compared with single drug group, berberine combined with olaparib significantly inhibited cell activity, and the difference was statistically significant $(\mathrm{P}<0.001)$.

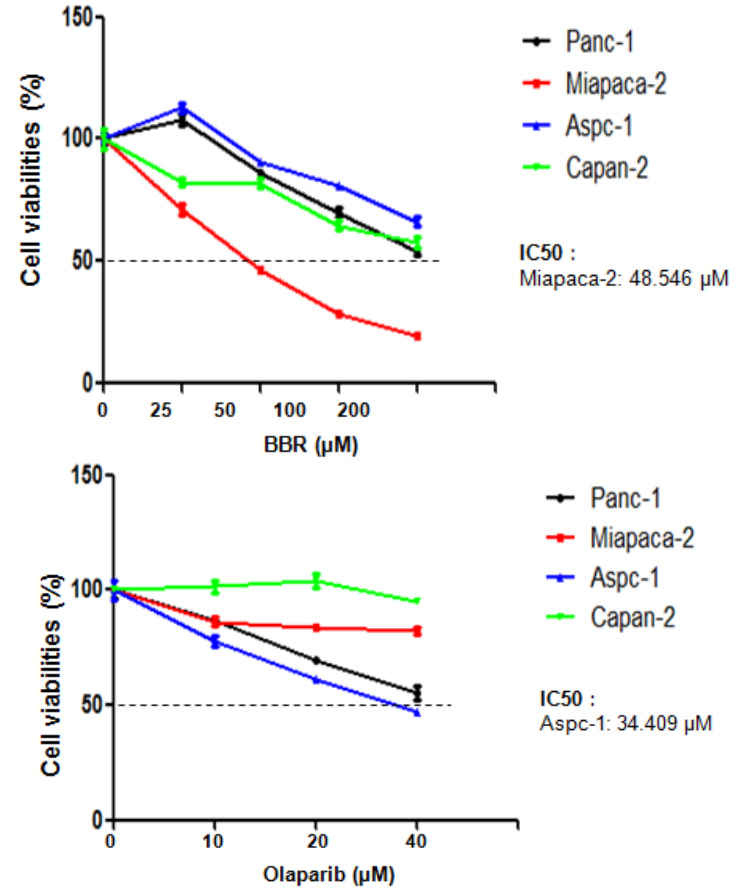

Figure 1 The inhibitory effect of berberine and olaparib alone on pancreatic cancer cell activity. 


\section{Effects of berberine, olaparib combined with z-VAD-FMK and Nec-1 on pancreatic cancer cell activity}

By CCK8 assay for cell activity, we proved that the combination of the two drugs could enhance cytotoxicity. Next, the apoptosis inhibitor z-VADFMK and the necrotic apoptosis inhibitor Nec-1 were combined respectively to verify the cell death pattern. Pancreatic

a

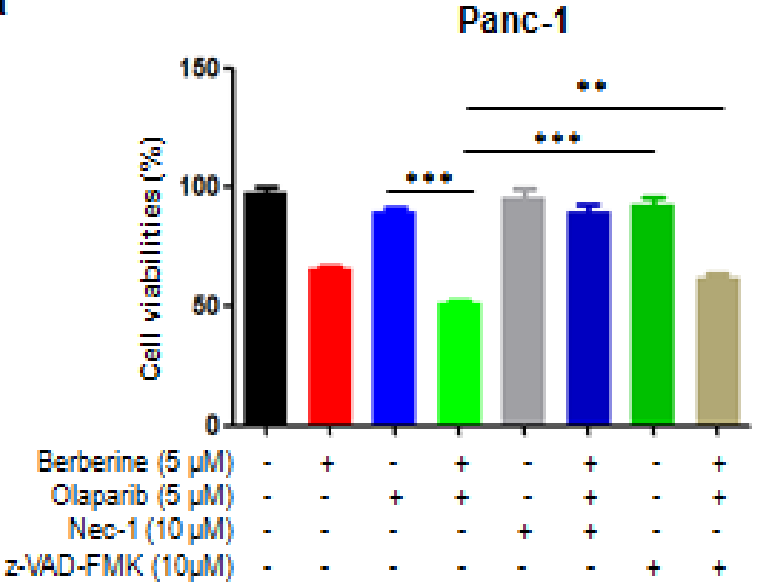

cancer cells Panc-1, Miapaca-2 and Aspc-1 were selected, As shown in Figure 2, The cytotoxic effect of berberine $(5 \mu \mathrm{M})$ combined with olaparib $(5 \mu \mathrm{M})$ was reversed by Nec-1. The difference was significant. The cytotoxicity could be partially reversed by z-VADFMK, and the difference was significant. The results showed that the two drugs had synergistic effect by inducing apoptosis.

b

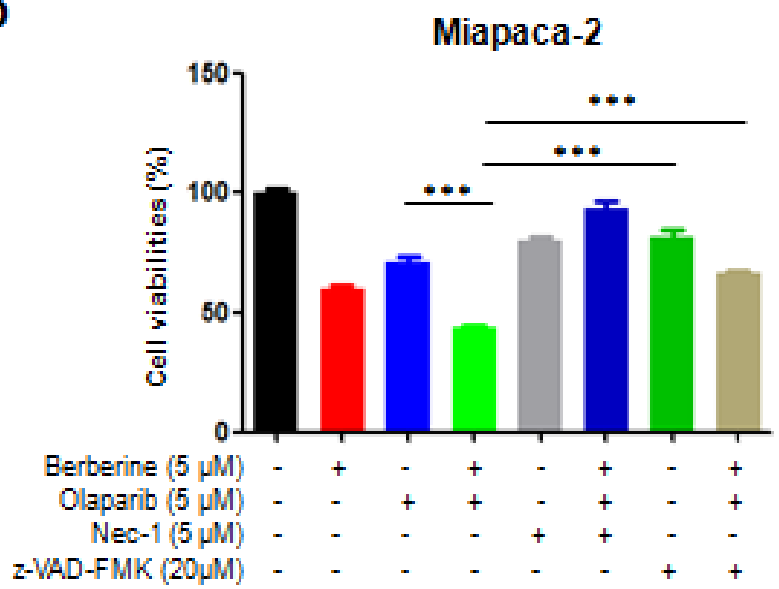

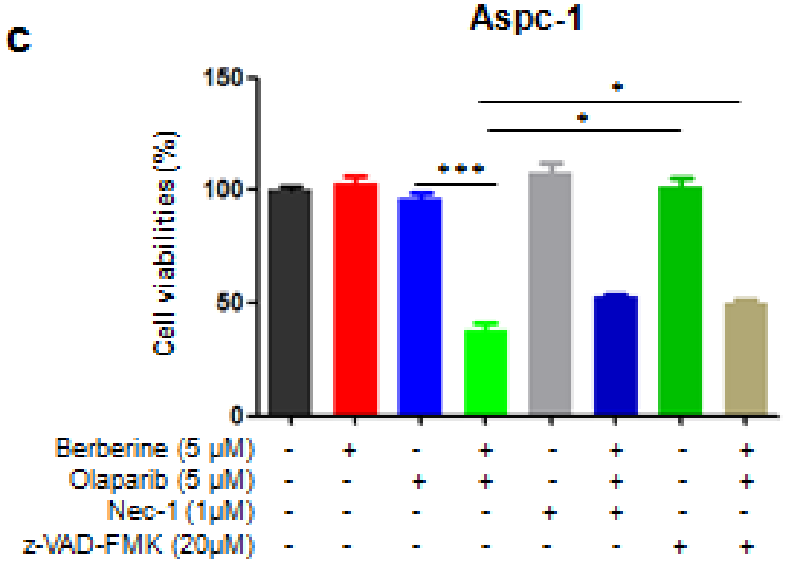

Figure 2 z-VADFMK and Nec-1 reversed the synergy between berberine and olaparib. * Means $\mathrm{P}<0.05$, ** means $\mathrm{P}<0.01, * * *$ means $\mathrm{P}<0.001$.

\section{Effects of berberine combined with olaparib on apoptosis}

Since the synergistic effect of berberine and olaparib on pancreatic cancer cells could be reversed by apoptosis inhibitor and necrosis inhibitor, we then verified this result by flow cytometry to detect apoptosis rate. As shown in Figure 3, induction of apoptosis by the two drugs in combination was far greater than olaparib treatment group $(\mathrm{p}<0.05)$. 
Panc-1

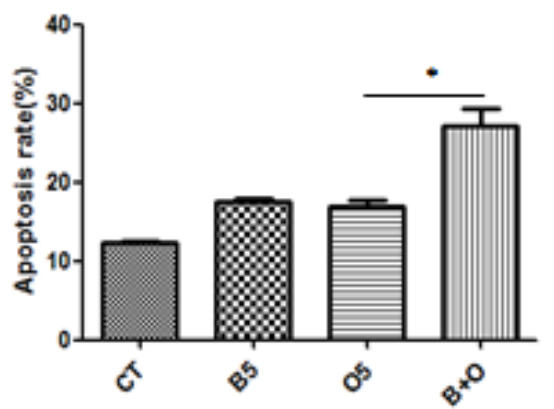

Miapaca-2

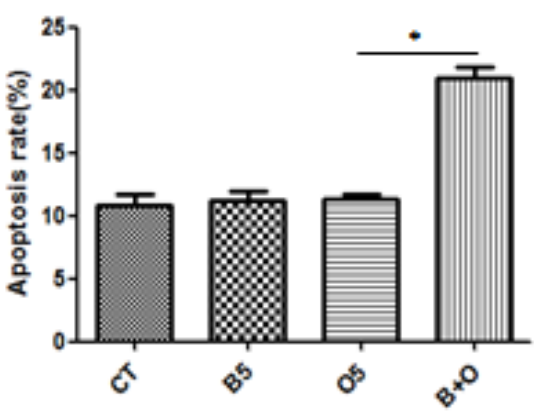

Aspc-1

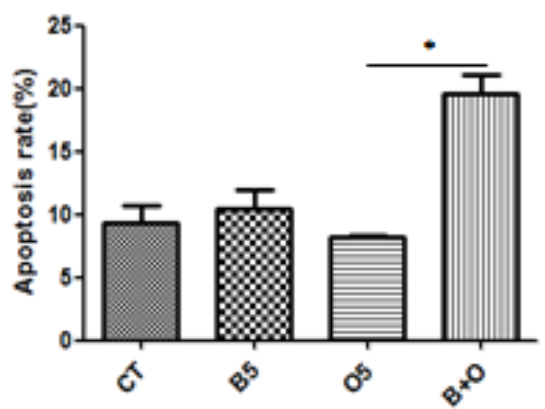

Figure 3 Berberine sensitizes pancreatic cancer cells to PARP inhibitor by induction of apoptosis. Panc-1 、 Miapaca-2 and Aspc-1 cells were treated with berberine or olaparib alone or in combination for $48 \mathrm{~h}$. Apoptosis was measured by flow cytometry with annexin $\mathrm{V}$ and PI staining. * means $\mathrm{P}<0.05$.

\section{Effects of berberine combined with olaparib on the expression levels of DNA damage repair related genes}

As shown in Figure 4, Rad51 expression was downregulated and Parp expression was up-regulated in berberine group and Rad51 expression was up-regulated

a

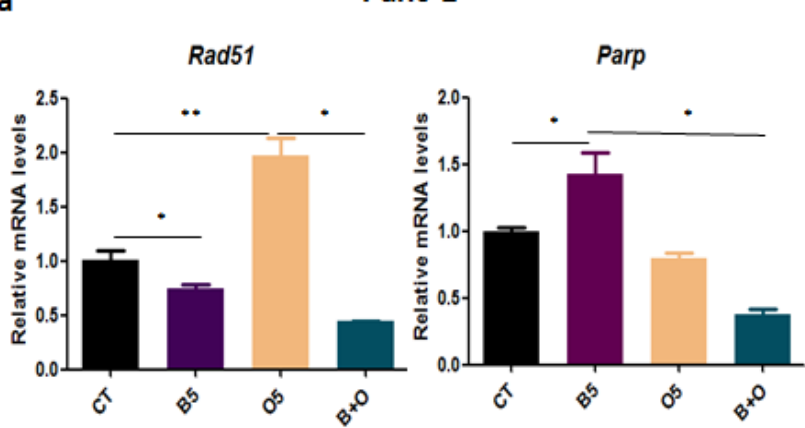

in olaparib group compared with the control group in Panc-1, Miapaca-2 and Aspc-1 cells. Compared with berberine treatment group, Parp expression was downregulated in the combination group. Compared with olaparib treatment group, Rad51 expression was downregulated in the combined treatment group. b

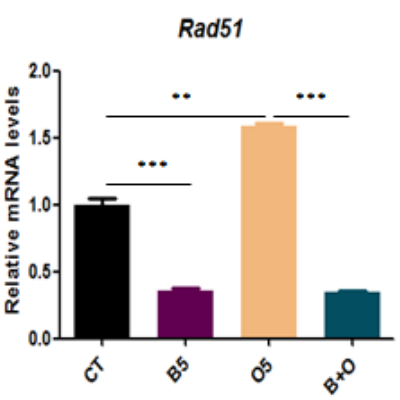

Aspc-1
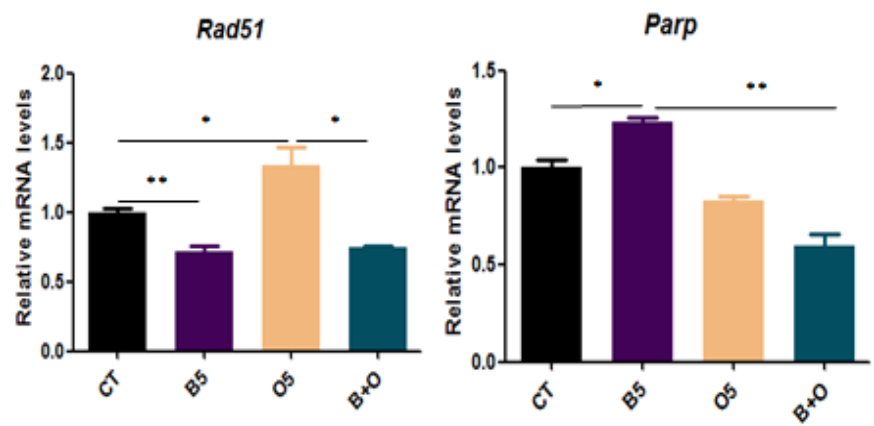

Figure 4 Berberine inhibits the expression of Rad51 and activates $P A R P$ in pancreatic cancer cells. In Panc-1(a), Miapaca-2(b) and Aspc-1(c) cells, Berberine downregulates Rad51 and activates PARP. When berberine is combined with olaparib, this trend can be reversed. * means $\mathrm{P}<0.05$, ** means $\mathrm{P}<0.01$, *** means $\mathrm{P}<0.001$.

\section{Discussion:}

Pancreatic cancer is a common malignancy of the digestive tract, with atypical early clinical symptoms, rapid progression and poor prognosis. The 5-year survival rate is less than $6 \%$. Due to the high degree of malignancy of pancreatic cancer, the bottleneck of diagnosis and treatment has been difficult to break through. ${ }^{[1]}$

PARP is a kind of protein-modifying enzyme in most eukaryotic cells, which plays an important role in DNA damage repair and genomic stability maintenance. PARP is the most critical factor for DNA single-stranded 
breaks (SSB) repair. When PARP activity is inhibited, a large number of unrepaired single-stranded gaps will lead to replication fork disintegration and turn into double-stranded gaps, that is DNA double-stranded breaks (DSB). ${ }^{[2]}$ Homologous recombination repair (HRR) is the main repair method of DNA double strand break. The main proteins involved in HRR include BRCA1, BRCA2 and "BRCA-like" proteins Rad51 and ATM, etc. However, when the function of these proteins is impaired or the gene is mutated, the HRR is not working properly, other DNA repair methods used by cells usually introduce large-scale genome recombination, resulting in cell death. ${ }^{[3]}$

At present, most of the studies on the inhibitory effect of PARP inhibitors on pancreatic cancer cells are focused on the combination with radiotherapy and chemotherapy drugs, such as Davide et al found that PARP inhibitor BSI-401, in conjunction with oxaliplatin, inhibited the growth of transplanted tumor in nude mice with pancreatic cancer cells. ${ }^{[4]}$ Richard et al studied the effect of PARP inhibitor ABT-888 combined with radiotherapy on pancreatic cancer cells Miapaca-2, and found that ABT- 888 combined with radiotherapy had a stronger inhibitory effect on pancreatic cancer cells than radiotherapy alone, and its inhibition effect was confirmed in transplanted tumors in nude mice. ${ }^{[5]}$ Jacob et al found that PARP inhibitor 3-ABA combined with gemcitabine was more toxic to pancreatic cancer cells and induced increased apoptosis. ${ }^{[6]}$

Berberine is a kind of medicine commonly used in the treatment of gastrointestinal diseases, and no serious side effects have been reported so far ${ }^{[7]}$. In breast cancer, ovarian cancer and esophageal squamous cell cancer, berberine can down-regulate the expression of homologous recombinant repair protein $\operatorname{Rad} 51$ and enhance the sensitivity of breast cancer and esophageal squamous cell cells to radiotherapy ${ }^{[8-9]}$. Whether berberine can inhibit the expression of $\operatorname{Rad} 51$ in pancreatic cancer cells and produce synthetic lethal effect by combining with PARP inhibitor has not been reported yet.

In this study, the combination of $5 \mu \mathrm{M}$ berberine and $5 \mu \mathrm{M}$ olaparib significantly inhibited the cell activity and significantly enhanced the sensitivity of pancreatic cancer cells to olaparib. The synergistic effect of berberine and olaparib can be reversed by apoptosis inhibitor and necrosis inhibitor. In pancreatic cancer cells, we demonstrated for the first time that berberine treatment could down-regulate the expression of Rad51, while after olaparib treatment, Rad51 expression was upregulated, which may be the reason for the resistance of pancreatic cancer cells to olaparib, and the high expression of Rad51 could be reversed by the combination of berberine. The up-regulated expression of Parp after berberine treatment indicated that Parp was activated. Since pancreatic cancer cells were more dependent on Parp and more sensitive to PARP inhibitors, the expression of Parp was significantly inhibited after combination with olaparib, which confirmed the above suggestion. Berberine can also upregulate Parp expression in ovarian cancer cells, and this activation can be reversed by PARP inhibitor niraparib
${ }^{[10]}$. In summary, berberine combined with olaparib may induce synthetic lethal effect on pancreatic cancer cells by inhibiting the expression of Rad51 and Parp, and induce apoptosis. In addition, how the combination of the two drugs affects the expression of Rad51 in pancreatic cancer cells and whether the combination of the two drugs on the basis of tumor formation in animals is consistent with the efficacy of in vitro experiments remains to be further verified.

\section{Reference:}

1. Sung Yong Han, et al."First-line chemotherapy in very elderly patients with metastatic pancreatic cancer: Gemcitabine monotherapy vs combination chemotherapy." World Journal of Clinical Cases 8.18(2020).

2. Dréan Amy, et al."PARP inhibitor combination therapy.." Critical reviews in oncology/hematology 108.(2016).

3. Plummer Elizabeth Ruth, and Calvert Hilary."Targeting poly(ADP-ribose) polymerase: a two-armed strategy for cancer therapy." Clinical cancer research : an official journal of the American Association for Cancer Research 13.21(2007).

4. Melisi Davide, et al."Oral poly(ADP-ribose) polymerase-1 inhibitor BSI-401 has antitumor activity and synergizes with oxaliplatin against pancreatic cancer, preventing acute neurotoxicity.." Clinical cancer research : an official journal of the American Association for Cancer Research 15.20(2009).

5. Richard Tuli, et al."Radiosensitization of Pancreatic Cancer Cells In Vitro and In Vivo through Poly (AD P-ribose) Polymerase Inhibition with ABT-888." Tr anslational Oncology 7.3(2014).

6. Dietmar A Jacob, et al."Combination therapy of pol y (ADP - ribose) polymerase inhibitor 3 - aminoben zamide and gemcitabine shows strong antitumor acti vity in pancreatic cancer cells." Journal of Gastroent erology and Hepatology 22.5(2007).

7. Chen Chunqiu, et al."Effects of berberine in the gastrointestinal tract - a review of actions and therapeutic implications.." The American journal of Chinese medicine 42.5(2014).

8. Jing Wang, et al."Radiosensitization effects of Berberine on human breast cancer cells." International Journal of Molecular Medicine 30.5(2012).

9. Qiao Liu, et al."Berberine radiosensitizes human esophageal cancer cells by downregulating homologous recombination repair protein RAD51.." PLoS ONE 6.8(2017).

10. Dong Hou, et al."Berberine induces oxidative DNA damage and impairs homologous recombination repair in ovarian cancer cells to confer increased sensitivity to PARP inhibition." Cell Death \& Disease 8.10(2017). 